\title{
Are Incentives for R\&D Effective? Evidence from a Regression Discontinuity Approach
}

\author{
Online Appendix
}

RAFFAELLO BRONZINI, AND ELEONORA IACHINI

DESCRIPTIVE STATISTICS OF THE SAMPLES USED IN THE REGRESSIONS

Table B1

\begin{tabular}{|c|c|c|c|c|c|c|c|c|c|c|c|c|}
\hline \multirow{2}{*}{ Variable } & \multicolumn{4}{|c|}{ All firms } & \multicolumn{4}{|c|}{ Small firms } & \multicolumn{4}{|c|}{ Large firms } \\
\hline & Min & Max & Median & Mean & Min & Max & Median & Mean & Min & Max & Median & Mean \\
\hline $\begin{array}{l}\text { Total investment/ } \\
\text { pre-program sales }\end{array}$ & -0.189 & 0.413 & 0.017 & 0.042 & -0.189 & 0.413 & 0.023 & 0.053 & -0.186 & 0.374 & 0.012 & 0.031 \\
\hline $\begin{array}{l}\text { Tangible investment / } \\
\text { pre-program sales }\end{array}$ & -0.372 & 0.471 & 0.005 & 0.027 & -0.336 & 0.471 & 0.003 & 0.027 & -0.372 & 0.354 & 0.008 & 0.026 \\
\hline $\begin{array}{l}\text { Intangible investment / } \\
\text { pre-program sales }\end{array}$ & -0.153 & 0.376 & 0.001 & 0.015 & -0.112 & 0.376 & 0.004 & 0.026 & -0.153 & 0.192 & 0.000 & 0.005 \\
\hline $\begin{array}{l}\text { Total investment/ } \\
\text { pre-program asset }\end{array}$ & -0.291 & 0.576 & 0.020 & 0.047 & -0.291 & 0.576 & 0.028 & 0.062 & -0.171 & 0.480 & 0.012 & 0.032 \\
\hline $\begin{array}{l}\text { Tangible investment / } \\
\text { pre-program asset }\end{array}$ & -0.316 & 0.707 & 0.005 & 0.032 & -0.316 & 0.707 & 0.004 & 0.033 & -0.153 & 0.484 & 0.008 & 0.030 \\
\hline $\begin{array}{l}\text { Intangible investment / } \\
\text { pre-program asset }\end{array}$ & -0.154 & 0.370 & 0.001 & 0.015 & -0.154 & 0.370 & 0.005 & 0.029 & -0.138 & 0.149 & 0.000 & 0.002 \\
\hline $\begin{array}{l}\text { Total investment/ } \\
\text { pre-program capital }\end{array}$ & -1.000 & 10.429 & 0.139 & 0.491 & -1.000 & 10.429 & 0.246 & 0.728 & -0.693 & 6.662 & 0.081 & 0.256 \\
\hline $\begin{array}{l}\text { Tangible investment / } \\
\text { pre-program capital }\end{array}$ & -0.749 & 6.504 & 0.046 & 0.266 & -0.749 & 6.504 & 0.046 & 0.339 & -0.604 & 3.438 & 0.051 & 0.194 \\
\hline $\begin{array}{l}\text { Intangible investment / } \\
\text { pre-program capital }\end{array}$ & -0.653 & 8.953 & 0.005 & 0.225 & -0.585 & 8.953 & 0.042 & 0.389 & -0.653 & 5.900 & -0.002 & 0.061 \\
\hline Total Investment & -103863 & 55137 & 80 & 769 & -986 & 2962 & 61 & 236 & -103863 & 55137 & 289 & 1300 \\
\hline Tangible investment & -43504 & 59262 & 29 & 895 & -1004 & 3156 & 13 & 146 & -43504 & 59262 & 160 & 1640 \\
\hline Intangible investment & -118504 & 35658 & 5 & -126 & -426 & 1905 & 17 & 90 & -118504 & 35658 & -9 & -340 \\
\hline $\begin{array}{l}\text { Labor costs/ } \\
\text { pre-program sales }\end{array}$ & 0 & 2.424 & 0.654 & 0.698 & 0.000 & 1.891 & 0.684 & 0.738 & 0.105 & 2.424 & 0.636 & 0.659 \\
\hline $\begin{array}{l}\text { Service costs/ } \\
\text { pre-program sales }\end{array}$ & 0.100 & 4.556 & 0.901 & 0.989 & 0.296 & 4.556 & 1.028 & 1.120 & 0.100 & 2.554 & 0.754 & 0.859 \\
\hline Log (Employment) & 1.386 & 10.040 & 5.394 & 5.625 & 1.386 & 5.497 & 4.564 & 4.484 & 4.443 & 10.040 & 5.967 & 6.189 \\
\hline Log (Wages) & 3.171 & 4.821 & 3.743 & 3.756 & 3.171 & 4.280 & 3.688 & 3.697 & 3.367 & 4.821 & 3.763 & 3.784 \\
\hline
\end{tabular}


RESULTS FOR EMPLOYMENT AND WAGES

\begin{tabular}{|c|c|c|c|c|c|c|}
\hline & \multicolumn{3}{|c|}{ Outcome variable: Log (Employment) } & \multicolumn{3}{|c|}{ Outcome variable: Log (Wages) } \\
\hline & All firms & Small firms & Large firms & All firms & Small firms & Large firms \\
\hline \multicolumn{7}{|c|}{$\begin{array}{l}\text { Panel A1. Full sample } \\
\text { Order of polynomial }\end{array}$} \\
\hline 0 & $\begin{array}{l}0.284 * \\
(0.154)\end{array}$ & $\begin{array}{l}0.226^{*} \\
(0.116)\end{array}$ & $\begin{array}{l}0.237 * \\
(0.129)\end{array}$ & $\begin{array}{c}0.056 * * \\
(0.023)\end{array}$ & $\begin{array}{l}-0.009 \\
(0.039)\end{array}$ & $\begin{array}{c}0.088 * * * \\
(0.027)\end{array}$ \\
\hline 1 & $\begin{array}{l}-0.096 \\
(0.228)\end{array}$ & $\begin{array}{c}0.278 \\
(0.167)\end{array}$ & $\begin{array}{l}-0.011 \\
(0.182)\end{array}$ & $\begin{array}{c}0.024 \\
(0.030)\end{array}$ & $\begin{array}{l}-0.026 \\
(0.054)\end{array}$ & $\begin{array}{c}0.065 \\
(0.040)\end{array}$ \\
\hline 2 & $\begin{array}{l}0.528 * \\
(0.277)\end{array}$ & $\begin{array}{l}0.808 * * * \\
(0.212)\end{array}$ & $\begin{array}{l}-0.158 \\
(0.265)\end{array}$ & $\begin{array}{c}0.031 \\
(0.041)\end{array}$ & $\begin{array}{l}-0.006 \\
(0.082)\end{array}$ & $\begin{array}{c}0.035 \\
(0.058)\end{array}$ \\
\hline 3 & $\begin{array}{c}0.377 \\
(0.339)\end{array}$ & $\begin{array}{c}0.326 \\
(0.337)\end{array}$ & $\begin{array}{l}-0.152 \\
(0.446)\end{array}$ & $\begin{array}{c}0.005 \\
(0.096)\end{array}$ & $\begin{array}{c}0.009 \\
(0.176)\end{array}$ & $\begin{array}{l}-0.013 \\
(0.126)\end{array}$ \\
\hline \multicolumn{7}{|c|}{$\begin{array}{l}\text { Panel A2. Local estimates: Wide-window sample } \\
\text { Order of polynomial }\end{array}$} \\
\hline 0 & $\begin{array}{c}0.077 \\
(0.191)\end{array}$ & $\begin{array}{c}0.331 * * \\
(0.143)\end{array}$ & $\begin{array}{l}-0.014 \\
(0.136)\end{array}$ & $\begin{array}{c}0.041 \\
(0.025)\end{array}$ & $\begin{array}{l}-0.016 \\
(0.054)\end{array}$ & $\begin{array}{c}0.069 * * * \\
(0.027)\end{array}$ \\
\hline 1 & $\begin{array}{c}0.407 \\
(0.318)\end{array}$ & $\begin{array}{l}0.725 * * * \\
(0.184)\end{array}$ & $\begin{array}{c}0.068 \\
(0.349)\end{array}$ & $\begin{array}{c}0.025 \\
(0.041)\end{array}$ & $\begin{array}{l}-0.027 \\
(0.053)\end{array}$ & $\begin{array}{c}0.044 \\
(0.064)\end{array}$ \\
\hline 2 & $\begin{array}{c}0.312 \\
(0.315)\end{array}$ & $\begin{array}{c}0.415 \\
(0.3289\end{array}$ & $\begin{array}{c}0.318 \\
(0.570)\end{array}$ & $\begin{array}{c}0.021 \\
(0.087)\end{array}$ & $\begin{array}{c}0.074 \\
(0.177)\end{array}$ & $\begin{array}{l}-0.001 \\
(0.139)\end{array}$ \\
\hline \multicolumn{7}{|c|}{$\begin{array}{l}\text { Notes: The table reports the differences of the outcome variable between recipient and non-recipient firms estimated at the cut-off score } \\
\text { (score }=75 \text { ). Employment is accumulated over the first } 3 \text { years after the assignment (including that of the assignment). Wages are calculated as } \\
\text { labor costs divided by employment accumulated over the same period. Polynomial of order } 0 \text { is the difference in mean between treated and } \\
\text { untreated. Small (large) firms are those with value added below (above) the median. In panel A1 the number of observations (firms) is } 263 \text {; in } \\
\text { panel A2 is } 118 \text {. } \\
*, * *, * * * \text { significant at } 10 \text { percent, } 5 \text { percent, } 1 \text { percent, respectively. }\end{array}$} \\
\hline
\end{tabular}


PRE-ASSIGNMENT MEAN-DIFFERENCES BY FIRMS' SIZE

(Standard errors in brackets)

\begin{tabular}{|c|c|c|c|c|c|c|}
\hline \multirow{2}{*}{ Variables } & \multicolumn{3}{|c|}{ Small Firms } & \multicolumn{3}{|c|}{ Large firms } \\
\hline & All & $\begin{array}{c}50 \text { percent } \\
\text { cut off } \\
\text { sample }\end{array}$ & $\begin{array}{c}35 \text { percent } \\
\text { cut off } \\
\text { sample }\end{array}$ & All & $\begin{array}{c}50 \text { percent } \\
\text { cut off } \\
\text { sample }\end{array}$ & $\begin{array}{c}35 \text { percent } \\
\text { cut off } \\
\text { sample }\end{array}$ \\
\hline Sales & $\begin{array}{l}1547 \\
(967)\end{array}$ & $\begin{array}{c}2534 \\
(1675)\end{array}$ & $\begin{array}{c}3364 \\
(2516)\end{array}$ & $\begin{array}{l}74782 * \\
(41275)\end{array}$ & $\begin{array}{c}3015 \\
(14429)\end{array}$ & $\begin{array}{c}10904 \\
(18833)\end{array}$ \\
\hline Value added & $\begin{array}{c}279 * * \\
(140)\end{array}$ & $\begin{array}{l}378 * \\
(194)\end{array}$ & $\begin{array}{c}392 \\
(258)\end{array}$ & $\begin{array}{c}16672 * \\
(9522)\end{array}$ & $\begin{array}{c}1612 \\
(3952)\end{array}$ & $\begin{array}{c}2801 \\
(5192)\end{array}$ \\
\hline Assets & $\begin{array}{c}654 \\
(634)\end{array}$ & $\begin{array}{l}1382 \\
(951)\end{array}$ & $\begin{array}{c}1392 \\
(1371)\end{array}$ & $\begin{array}{l}65424 * \\
(35288)\end{array}$ & $\begin{array}{c}7686 \\
(15092)\end{array}$ & $\begin{array}{c}12096 \\
(19549)\end{array}$ \\
\hline ROA & $\begin{array}{c}2.85 \\
(1.96)\end{array}$ & $\begin{array}{c}3.16 \\
(2.18)\end{array}$ & $\begin{array}{c}3.30 \\
(2.14)\end{array}$ & $\begin{array}{l}-1.36 \\
(1.23)\end{array}$ & $\begin{array}{l}-2.52 \\
(1.72)\end{array}$ & $\begin{array}{c}-0.59 \\
(1.60)\end{array}$ \\
\hline Own capital/Debts & $\begin{array}{c}-0.017 \\
(0.088)\end{array}$ & $\begin{array}{c}-0.176^{*} \\
(0.104)\end{array}$ & $\begin{array}{c}-0.137 \\
(0.120)\end{array}$ & $\begin{array}{c}-0.136 \\
(0.143)\end{array}$ & $\begin{array}{c}-0.268 \\
(0.212)\end{array}$ & $\begin{array}{c}-0.341 \\
(0.281)\end{array}$ \\
\hline Gross operating margin/Sales & $\begin{array}{c}0.024 \\
(0.015)\end{array}$ & $\begin{array}{c}0.021 \\
(0.019)\end{array}$ & $\begin{array}{c}0.005 \\
(0.022)\end{array}$ & $\begin{array}{l}-0.005 \\
(0.012)\end{array}$ & $\begin{array}{l}-0.020 \\
(0.017)\end{array}$ & $\begin{array}{c}-0.012 \\
(0.017)\end{array}$ \\
\hline Cash flow/Sales & $\begin{array}{c}0.025^{* *} * \\
(0.011)\end{array}$ & $\begin{array}{c}0.023 \\
(0.017)\end{array}$ & $\begin{array}{c}0.022 \\
(0.023)\end{array}$ & $\begin{array}{c}0.008 \\
(0.013)\end{array}$ & $\begin{array}{c}-0.006 \\
(0.013)\end{array}$ & $\begin{array}{c}0.002 \\
(0.012)\end{array}$ \\
\hline Financial costs/Debts & $\begin{array}{c}0.001 \\
(0.002)\end{array}$ & $\begin{array}{c}0.001 \\
(0.003)\end{array}$ & $\begin{array}{c}0.000 \\
(0.003)\end{array}$ & $\begin{array}{c}-0.014 \\
(0.009)\end{array}$ & $\begin{array}{c}-0.014 \\
(0.016)\end{array}$ & $\begin{array}{c}-0.014 \\
(0.023)\end{array}$ \\
\hline Labor costs/Sales & $\begin{array}{c}-0.005 \\
(0.015)\end{array}$ & $\begin{array}{c}-0.012 \\
(0.022)\end{array}$ & $\begin{array}{c}-0.031 \\
(0.030)\end{array}$ & $\begin{array}{c}-0.008 \\
(0.014)\end{array}$ & $\begin{array}{c}0.021 \\
(0.019)\end{array}$ & $\begin{array}{c}-0.001 \\
(0.023)\end{array}$ \\
\hline Service costs/Sales & $\begin{array}{c}-0.025 \\
(0.020)\end{array}$ & $\begin{array}{c}-0.007 \\
(0.026)\end{array}$ & $\begin{array}{c}0.007 \\
(0.032)\end{array}$ & $\begin{array}{l}0.0165 \\
(0.018)\end{array}$ & $\begin{array}{c}0.045 * * \\
(0.019)\end{array}$ & $\begin{array}{c}0.051 * * \\
(0.024)\end{array}$ \\
\hline Total investment/ Sales & $\begin{array}{c}0.007 \\
(0.014)\end{array}$ & $\begin{array}{c}0.027 \\
(0.025)\end{array}$ & $\begin{array}{c}0.053 \\
(0.034)\end{array}$ & $\begin{array}{c}-0.004 \\
(0.012)\end{array}$ & $\begin{array}{c}-0.013 \\
(0.015)\end{array}$ & $\begin{array}{c}-0.007 \\
(0.017)\end{array}$ \\
\hline Tangible investment/Sales & $\begin{array}{c}0.017 \\
(0.013)\end{array}$ & $\begin{array}{c}0.035 \\
(0.022)\end{array}$ & $\begin{array}{c}0.051 \\
(0.032)\end{array}$ & $\begin{array}{c}0.006 \\
(0.012)\end{array}$ & $\begin{array}{c}0.003 \\
(0.020)\end{array}$ & $\begin{array}{c}0.014 \\
(0.025)\end{array}$ \\
\hline Intangible investment/Sales & $\begin{array}{c}-0.011 * * \\
(0.005)\end{array}$ & $\begin{array}{c}-0.008 \\
(0.008)\end{array}$ & $\begin{array}{c}0.003 \\
(0.009)\end{array}$ & $\begin{array}{l}-0.010 \\
(0.012)\end{array}$ & $\begin{array}{c}-0.016 \\
(0.017)\end{array}$ & $\begin{array}{l}-0.021 \\
(0.022)\end{array}$ \\
\hline Number of firms & 178 & 90 & 58 & 179 & 81 & 57 \\
\hline
\end{tabular}

$*, * *, * * *$ : significant at 10 percent, 5 percent, 1 percent, respectively. 
Table B4

EFFECT OF THE PROGRAM ON DIFFERENT OUTCOME VARIABLES BY FIRMS’ SIZE

\begin{tabular}{|c|c|c|c|c|c|c|}
\hline & \multicolumn{3}{|c|}{ Labor costs/ Pre-program sales } & \multicolumn{3}{|c|}{ Service costs/ Pre-program sales } \\
\hline & Small & Large & AIC & Small & Large & AIC \\
\hline \multicolumn{7}{|c|}{$\begin{array}{l}\text { Panel A. Full sample } \\
\text { Order of polynomial }\end{array}$} \\
\hline 0 & $\begin{array}{l}-0.001 \\
(0.064)\end{array}$ & $\begin{array}{l}-0.093 \\
(0.086)\end{array}$ & 242.4 & $\begin{array}{l}-0.069 \\
(0.085)\end{array}$ & $\begin{array}{l}-0.057 \\
(0.089)\end{array}$ & 527.9 \\
\hline 1 & $\begin{array}{l}-0.068 \\
(0.095)\end{array}$ & $\begin{array}{l}-0.041 \\
(0.138)\end{array}$ & 248.5 & $\begin{array}{c}0.026 \\
(0.137)\end{array}$ & $\begin{array}{c}0.031 \\
(0.136)\end{array}$ & 533.5 \\
\hline 2 & $\begin{array}{l}-0.069 \\
(0.118)\end{array}$ & $\begin{array}{l}-0.241 \\
(0.171)\end{array}$ & 249.9 & $\begin{array}{c}0.076 \\
(0.181)\end{array}$ & $\begin{array}{l}-0.079 \\
(0.188)\end{array}$ & 540.4 \\
\hline 3 & $\begin{array}{l}-0.247 \\
(0.156)\end{array}$ & $\begin{array}{l}-0.625^{*} \\
(0.348)\end{array}$ & 241.8 & $\begin{array}{c}0.220 \\
(0.185)\end{array}$ & $\begin{array}{r}-0.604^{*} \\
(0.313)\end{array}$ & 541.1 \\
\hline \multicolumn{7}{|c|}{$\begin{array}{l}\text { Panel B. Local estimates: Wide-window sample } \\
\text { Order of polynomial }\end{array}$} \\
\hline 0 & $\begin{array}{c}0.004 \\
(0.096)\end{array}$ & $\begin{array}{l}-0.010 \\
(0.097)\end{array}$ & 134.2 & $\begin{array}{l}-0.013 \\
(0.116)\end{array}$ & $\begin{array}{c}0.018 \\
(0.091)\end{array}$ & 256.6 \\
\hline 1 & $\begin{array}{c}-0.262 * * \\
(0.115)\end{array}$ & $\begin{array}{l}-0.290^{*} \\
(0.155)\end{array}$ & 125.8 & $\begin{array}{c}0.062 \\
(0.195)\end{array}$ & $\begin{array}{l}-0.201 \\
(0.167)\end{array}$ & 262.5 \\
\hline 2 & $\begin{array}{l}-0.049 \\
(0.149)\end{array}$ & $\begin{array}{l}-0.206 \\
(0.256)\end{array}$ & 127.3 & $\begin{array}{c}0.246 \\
(0.200)\end{array}$ & $\begin{array}{l}-0.155 \\
(0.275)\end{array}$ & 267.2 \\
\hline \multicolumn{7}{|c|}{$\begin{array}{l}\text { Panel C. Local estimates: Narrow- window sample } \\
\text { Order of polynomial }\end{array}$} \\
\hline 0 & $\begin{array}{l}-0.066 \\
(0.102)\end{array}$ & $\begin{array}{l}-0.121 \\
(0.110)\end{array}$ & 94.6 & $\begin{array}{c}0.021 \\
(0.166)\end{array}$ & $\begin{array}{l}-0.057 \\
(0.109)\end{array}$ & 194.1 \\
\hline 1 & $\begin{array}{l}-0.215 \\
(0.135)\end{array}$ & $\begin{array}{l}-0.238 \\
(0.245)\end{array}$ & 96.2 & $\begin{array}{c}0.256 \\
(0.288)\end{array}$ & $\begin{array}{l}-0.179 \\
(0.257)\end{array}$ & 198.5 \\
\hline 2 & $\begin{array}{c}0.340 * * \\
(0.122)\end{array}$ & $\begin{array}{l}-0.009 \\
(0.354)\end{array}$ & 93.4 & $\begin{array}{c}0.209 \\
(0.342)\end{array}$ & $\begin{array}{l}-0.226 \\
(0.316)\end{array}$ & 191.5 \\
\hline
\end{tabular}

Notes: The table shows the estimates of the coefficient $\beta_{k}$ of model (2) using labor and services costs scaled by the preassignment sales. Costs are accumulated over the first 3 years after the assignment (included that of the assignment). Robust standard errors clustered by score are in round brackets. AIC is the Akaike Information Criterion. Small [Large] firms are those falling in the first [second] half of the distribution of the value added. Number of observations (firms) is 357 in Panel A; 171 in Panel B; 115 in Panel C.

$*, * *, * * *$ significant at 10 percent, 5 percent, 1 percent, respectively. 
Table B5

RESULTS FOR SERVICES

Outcome variable: Total investment/Pre-program sales

\begin{tabular}{|c|c|c|c|c|c|}
\hline & \multicolumn{2}{|c|}{ Model (1) } & \multicolumn{3}{|c|}{ Model (2) } \\
\hline & $\beta$ & AIC & $\beta$ - Small & $\beta$ - Large & AIC \\
\hline \multicolumn{6}{|l|}{$\begin{array}{l}\text { Panel A. Full sample } \\
\text { Order of polynomial }\end{array}$} \\
\hline 0 & $\begin{array}{c}0.032 \\
(0.025)\end{array}$ & -86.5 & $\begin{array}{l}0.068 * \\
(0.036)\end{array}$ & $\begin{array}{c}0.000 \\
(0.036)\end{array}$ & -85.2 \\
\hline 1 & $\begin{array}{l}-0.016 \\
(0.036)\end{array}$ & -85.2 & $\begin{array}{c}0.048 \\
(0.046)\end{array}$ & $\begin{array}{l}-0.114 \\
(0.032)\end{array}$ & -83.1 \\
\hline 2 & $\begin{array}{c}0.036 \\
(0.050)\end{array}$ & -82.9 & $\begin{array}{l}0.139 * * * \\
(0.044)\end{array}$ & $\begin{array}{l}-0.085 \\
(0.054)\end{array}$ & -77.6 \\
\hline 3 & $\begin{array}{c}0.034 \\
(0.091)\end{array}$ & -80.6 & $\begin{array}{l}0.191^{*} \\
(0.099)\end{array}$ & $\begin{array}{l}-0.165 \\
(0.122)\end{array}$ & -72.5 \\
\hline \multicolumn{6}{|c|}{$\begin{array}{l}\text { Panel B. Local estimates: Wide-window sample } \\
\text { Order of polynomial }\end{array}$} \\
\hline 0 & $\begin{array}{c}0.030 \\
(0.032)\end{array}$ & -66.4 & $\begin{array}{l}0.074 * \\
(0.042)\end{array}$ & $\begin{array}{l}-0.055^{*} \\
(0.031)\end{array}$ & -67.5 \\
\hline 1 & $\begin{array}{l}-0.035 \\
(0.040)\end{array}$ & -64.9 & $\begin{array}{c}0.052 \\
(0.047)\end{array}$ & $\begin{array}{c}-0.126 * * * \\
(0.031)\end{array}$ & -62.8 \\
\hline 2 & $\begin{array}{c}0.057 \\
(0.074)\end{array}$ & -63.7 & $\begin{array}{c}0.224 * * \\
(0.090)\end{array}$ & $\begin{array}{c}-0.083 \\
(0.087)\end{array}$ & -60.6 \\
\hline $\begin{array}{l}\text { Mean (st. dev.) for } \\
\text { untreated firms - Full } \\
\text { sample }\end{array}$ & $\begin{array}{c}0.030 \\
(0.143)\end{array}$ & & $\begin{array}{c}0.029 \\
(0.158)\end{array}$ & $\begin{array}{c}0.031 \\
(0.127)\end{array}$ & \\
\hline
\end{tabular}

Notes: The table shows the estimates of the coefficient $\beta$ of model (1) and (2) on service firms. For further details see the notes to Tables 3 and 5. Number of observations (firms) is 111 in Panel A; 67 in Panel B.

$*, * *, * * *$ : significant at 10 percent, 5 percent and 1 percent, respectively. 
ROBUSTNESS: ESTIMATIONS WITH COVARIATES

Outcome variable: Total investment/Pre-program sales

\begin{tabular}{|c|c|c|c|c|c|}
\hline & \multicolumn{2}{|c|}{ Model $(1)+$ covariates } & \multicolumn{3}{|c|}{ Model $(2)+$ covariates } \\
\hline & $\beta$ & AIC & $\beta$ - Small & $\beta$ - Large & AIC \\
\hline \multicolumn{6}{|c|}{$\begin{array}{l}\text { Panel A. Full sample } \\
\text { Order of polynomial }\end{array}$} \\
\hline 0 & $\begin{array}{c}0.015 \\
(0.012)\end{array}$ & -585.9 & $\begin{array}{c}0.041^{* *} \\
(0.016)\end{array}$ & $\begin{array}{l}-0.015 \\
(0.018)\end{array}$ & -589.54 \\
\hline 1 & $\begin{array}{l}0.036^{*} \\
(0.019)\end{array}$ & -584.2 & $\begin{array}{c}0.071 * * * \\
(0.026)\end{array}$ & $\begin{array}{l}-0.009 \\
(0.025)\end{array}$ & -584.4 \\
\hline 2 & $\begin{array}{c}0.038 \\
(0.029)\end{array}$ & -581.9 & $\begin{array}{c}0.090^{* * *} \\
(0.031)\end{array}$ & $\begin{array}{c}-0.016 \\
(0.038)\end{array}$ & -578.9 \\
\hline 3 & $\begin{array}{c}0.064 \\
(0.040)\end{array}$ & -579.2 & $\begin{array}{l}0.142 * * * \\
(0.043)\end{array}$ & $\begin{array}{l}-0.024 \\
(0.061)\end{array}$ & -575.9 \\
\hline \multicolumn{6}{|c|}{$\begin{array}{l}\text { Panel B. Local estimates: Wide-window sample } \\
\text { Order of polynomial }\end{array}$} \\
\hline 0 & $\begin{array}{c}0.021 \\
(0.018)\end{array}$ & -267.1 & $\begin{array}{l}0.050^{*} \\
(0.025)\end{array}$ & $\begin{array}{l}-0.013 \\
(0.022)\end{array}$ & -266.8 \\
\hline 1 & $\begin{array}{c}0.034 \\
(0.037)\end{array}$ & -263.4 & $\begin{array}{c}0.084 * * \\
(0.039)\end{array}$ & $\begin{array}{l}-0.008 \\
(0.004)\end{array}$ & -264.1 \\
\hline 2 & $\begin{array}{l}0.101^{*} \\
(0.053)\end{array}$ & -263.8 & $\begin{array}{c}0.165 * * * \\
(0.057)\end{array}$ & $\begin{array}{c}0.042 \\
(0.081)\end{array}$ & -265.5 \\
\hline \multicolumn{6}{|c|}{$\begin{array}{l}\text { Panel C. Local estimates: Narrow-window sample } \\
\text { Order of polynomial }\end{array}$} \\
\hline 0 & $\begin{array}{c}0.035 \\
(0.022)\end{array}$ & -189.1 & $\begin{array}{l}0.064 * * \\
(0.028)\end{array}$ & $\begin{array}{c}0.001 \\
(0.026)\end{array}$ & -193.2 \\
\hline 1 & $\begin{array}{c}0.062 \\
(0.044)\end{array}$ & -190.1 & $\begin{array}{c}0.143 * * \\
(0.059)\end{array}$ & $\begin{array}{l}-0.011 \\
(0.062)\end{array}$ & -196.9 \\
\hline 2 & $\begin{array}{l}-0.066 \\
(0.040)\end{array}$ & -193.8 & $\begin{array}{c}0.038 \\
(0.049)\end{array}$ & $\begin{array}{l}-0.186^{*} \\
(0.093)\end{array}$ & -202.9 \\
\hline
\end{tabular}

Notes: The table shows the estimates of the coefficient $\beta$ of model (1) and (2) on industrial firms including as covariates 2digit sector dummies, gross operative margin/value added, own capital/debts, ROA, cash flow/sales, total assets, financial costs/debts all referred to the pre-treatment period. Number of observations (firms) is 357 in Panel A; 171 in Panel B; 115 in Panel C.

*,**,***: significant at 10 percent, 5 percent and 1 percent, respectively. 
Table B7

EFFECT OF THE PROGRAM ON NON-NORMALIZED INVESTMENT

\begin{tabular}{|c|c|c|c|c|c|c|}
\hline & \multicolumn{3}{|c|}{ Total investment } & \multicolumn{3}{|c|}{ Log (Total investment) } \\
\hline & All firms & Small & Large & All firms & Small & Large \\
\hline \multicolumn{7}{|c|}{$\begin{array}{l}\text { Panel A. Full sample } \\
\text { Order of polynomial }\end{array}$} \\
\hline 0 & $\begin{array}{c}421.5 \\
(756.9)\end{array}$ & $\begin{array}{l}192.5 * \\
(104.7)\end{array}$ & $\begin{array}{c}456.9 \\
(1459.3)\end{array}$ & $\begin{array}{l}-0.039 \\
(0.045)\end{array}$ & $\begin{array}{l}0.002 * \\
(0.001)\end{array}$ & $\begin{array}{l}-0.078 \\
(0.085)\end{array}$ \\
\hline 1 & $\begin{array}{l}-154.7 \\
(641.9)\end{array}$ & $\begin{array}{c}419.8 * * * \\
(112.3)\end{array}$ & $\begin{array}{c}-780.9 \\
(1208.4)\end{array}$ & $\begin{array}{l}-0.002 \\
(0.011)\end{array}$ & $\begin{array}{c}0.004 * * * \\
(0.001)\end{array}$ & $\begin{array}{l}-0.022 \\
(0.028)\end{array}$ \\
\hline 2 & $\begin{array}{c}301.2 \\
(1102.9)\end{array}$ & $\begin{array}{c}338.5 * * \\
(151.8)\end{array}$ & $\begin{array}{c}209.9 \\
(2379.5)\end{array}$ & $\begin{array}{c}0.058 \\
(0.061)\end{array}$ & $\begin{array}{c}0.003 * * \\
(0.001)\end{array}$ & $\begin{array}{c}0.131 \\
(0.136)\end{array}$ \\
\hline 3 & $\begin{array}{c}1450.9 \\
(1346.7)\end{array}$ & $\begin{array}{c}584.2 * * * \\
(186.3)\end{array}$ & $\begin{array}{c}3585.5 \\
(2787.9)\end{array}$ & $\begin{array}{c}0.002 \\
(0.022)\end{array}$ & $\begin{array}{c}0.005^{* * *} * \\
(0.001)\end{array}$ & $\begin{array}{c}0.024 \\
(0.057)\end{array}$ \\
\hline \multicolumn{7}{|c|}{$\begin{array}{l}\text { Panel B. Local estimates: Wide-window sample } \\
\text { Order of polynomial }\end{array}$} \\
\hline 0 & $\begin{array}{c}326.9 \\
(477.1)\end{array}$ & $\begin{array}{l}319.0 * * * \\
(111.9)\end{array}$ & $\begin{array}{c}264.4 \\
(873.9)\end{array}$ & $\begin{array}{c}0.002 \\
(0.003)\end{array}$ & $\begin{array}{l}0.003 * * * \\
(0.001)\end{array}$ & $\begin{array}{c}0.001 \\
(0.007)\end{array}$ \\
\hline 1 & $\begin{array}{c}644.8 \\
(904.2)\end{array}$ & $\begin{array}{l}363.5^{* *} \\
(163.7)\end{array}$ & $\begin{array}{c}1363.5 \\
(1344.9)\end{array}$ & $\begin{array}{c}0.004 \\
(0.007)\end{array}$ & $\begin{array}{c}0.003 * * \\
(0.002)\end{array}$ & $\begin{array}{c}0.009 \\
(0.011)\end{array}$ \\
\hline 2 & $\begin{array}{c}913.0 \\
(954.9)\end{array}$ & $\begin{array}{c}685.9 * * \\
(247.4)\end{array}$ & $\begin{array}{c}2187.7 \\
(2152.9)\end{array}$ & $\begin{array}{c}0.008 \\
(0.009)\end{array}$ & $\begin{array}{c}0.007 * * * \\
(0.020)\end{array}$ & $\begin{array}{c}0.020 \\
(0.020)\end{array}$ \\
\hline \multicolumn{7}{|c|}{$\begin{array}{l}\text { Panel C. Local estimates: Narrow-window sample } \\
\text { Order of polynomial }\end{array}$} \\
\hline 0 & $\begin{array}{c}614.5 \\
(560.4)\end{array}$ & $\begin{array}{l}275.2 * \\
(143.7)\end{array}$ & $\begin{array}{c}886.9 \\
(740.3)\end{array}$ & $\begin{array}{c}0.004 \\
(0.004)\end{array}$ & $\begin{array}{l}0.003 * \\
(0.001)\end{array}$ & $\begin{array}{c}0.006 \\
(0.006)\end{array}$ \\
\hline 1 & $\begin{array}{c}679.5 \\
(891.1)\end{array}$ & $\begin{array}{l}723.2 * * \\
(308.9)\end{array}$ & $\begin{array}{c}1177.8 \\
(1626.6)\end{array}$ & $\begin{array}{c}0.005 \\
(0.008)\end{array}$ & $\begin{array}{c}0.007 * * \\
(0.003)\end{array}$ & $\begin{array}{c}0.009 \\
(0.014)\end{array}$ \\
\hline 2 & $\begin{array}{c}-3413 \\
(841.5)\end{array}$ & $\begin{array}{l}-123.6 \\
(325.2)\end{array}$ & $\begin{array}{c}-6897 * * * \\
(1412.1)\end{array}$ & $\begin{array}{c}-0.032 * * * \\
(0.007)\end{array}$ & $\begin{array}{l}-0.001 \\
(0.003)\end{array}$ & $\begin{array}{c}-0.063 * * * \\
(0.012)\end{array}$ \\
\hline
\end{tabular}

Notes: The table shows the estimates of the coefficient $\beta$ of model (1) and (2) using different outcome variables. Number of observations (firms) is 357 in Panel A; 171 in Panel B; 115 in Panel C. Since investment can be negative to calculate log of investment over the same sample used in the baseline regression we added $(1+$ the minimum of investment); i.e. the dependent variable is: $\log [$ investment $+1+\min ($ investment)]; where min(investment) is the minimum of the investment across firms. See the notes to table 5 for further details. 
EFFECT OF THE PROGRAM ON INVESTMENT NORMALIZED BY PRE-PROGRAM CAPITAL

\begin{tabular}{|c|c|c|c|c|c|c|c|c|c|}
\hline & \multicolumn{3}{|c|}{ Total investment/ Total pre-program capital } & \multicolumn{3}{|c|}{ Tangible investment/ Total pre-program capital } & \multicolumn{3}{|c|}{ Intangible investment/ Total pre-program capital } \\
\hline & All firms & Small & Large & All firms & Small & Large & All firms & Small & Large \\
\hline \multicolumn{10}{|c|}{$\begin{array}{l}\text { Panel A. Full sample } \\
\text { Order of polynomial }\end{array}$} \\
\hline 0 & $\begin{array}{c}0.192 \\
(0.135)\end{array}$ & $\begin{array}{l}0.432^{*} \\
(0.233)\end{array}$ & $\begin{array}{c}0.021 \\
(0.110)\end{array}$ & $\begin{array}{c}0.089 \\
(0.081)\end{array}$ & $\begin{array}{c}0.186 \\
(0.144)\end{array}$ & $\begin{array}{c}0.010 \\
(0.081)\end{array}$ & $\begin{array}{c}0.102 \\
(0.099)\end{array}$ & $\begin{array}{c}0.245 \\
(0.144)\end{array}$ & $\begin{array}{c}0.011 \\
(0.062)\end{array}$ \\
\hline 1 & $\begin{array}{c}0.470 \\
(0.236)\end{array}$ & $\begin{array}{l}0.751^{*} \\
(0.381)\end{array}$ & $\begin{array}{c}0.138 \\
(0.201)\end{array}$ & $\begin{array}{c}0.137 \\
(0.126)\end{array}$ & $\begin{array}{c}0.208 \\
(0.226)\end{array}$ & $\begin{array}{c}0.007 \\
(0.112)\end{array}$ & $\begin{array}{l}0.332 * \\
(0.179)\end{array}$ & $\begin{array}{r}0.543 * * \\
(0.237)\end{array}$ & $\begin{array}{c}0.130 \\
(0.142)\end{array}$ \\
\hline 2 & $\begin{array}{c}0.658^{* *} \\
(0.314)\end{array}$ & $\begin{array}{c}1.266^{* * *} \\
(0.443)\end{array}$ & $\begin{array}{c}0.019 \\
(0.264)\end{array}$ & $\begin{array}{c}0.130 \\
(0.183)\end{array}$ & $\begin{array}{c}0.383 \\
(0.284)\end{array}$ & $\begin{array}{l}-0.111 \\
(0.159)\end{array}$ & $\begin{array}{c}0.528^{* *} \\
(0.212)\end{array}$ & $\begin{array}{l}0.882 * * * \\
(0.271)\end{array}$ & $\begin{array}{c}0.131 \\
(0.172)\end{array}$ \\
\hline 3 & $\begin{array}{c}1.083 * * * \\
(0.341)\end{array}$ & $\begin{array}{c}2.089 * * * \\
(0.378)\end{array}$ & $\begin{array}{l}-0.425 \\
(0.302)\end{array}$ & $\begin{array}{l}0.365^{*} \\
(0.209)\end{array}$ & $\begin{array}{l}0.894 * * * \\
(0.239)\end{array}$ & $\begin{array}{l}-0.360 \\
(0.212)\end{array}$ & $\begin{array}{l}0.718^{* *} \\
(0.217)\end{array}$ & $\begin{array}{l}1.194 * * * \\
(0.281)\end{array}$ & $\begin{array}{l}-0.065 \\
(0.182)\end{array}$ \\
\hline \multicolumn{10}{|c|}{$\begin{array}{l}\text { Panel B. Local estimates: Wide-window sample } \\
\text { Order of polynomial }\end{array}$} \\
\hline 0 & $\begin{array}{l}0.429 * \\
(0.215)\end{array}$ & $\begin{array}{r}0.718^{* *} \\
(0.321)\end{array}$ & $\begin{array}{c}0.145 \\
(0.195)\end{array}$ & $\begin{array}{c}0.094 \\
(0.109)\end{array}$ & $\begin{array}{c}0.196 \\
(0.205)\end{array}$ & $\begin{array}{l}-0.012 \\
(0.088)\end{array}$ & $\begin{array}{l}0.336^{*} \\
(0.179)\end{array}$ & $\begin{array}{l}0.522 * * \\
(0.222)\end{array}$ & $\begin{array}{c}0.157 \\
(0.161)\end{array}$ \\
\hline 1 & $\begin{array}{c}0.562 \\
(0.412)\end{array}$ & $\begin{array}{l}1.306^{* *} \\
(0.494)\end{array}$ & $\begin{array}{l}-0.316 \\
(0.273)\end{array}$ & $\begin{array}{l}0.205 \\
(0.204)\end{array}$ & $\begin{array}{c}0.504 \\
(0.298)\end{array}$ & $\begin{array}{l}-0.087 \\
(0.148)\end{array}$ & $\begin{array}{c}0.358 \\
(0.259)\end{array}$ & $\begin{array}{c}0.801 * * * \\
(0.281)\end{array}$ & $\begin{array}{l}-0.229 \\
(0.175)\end{array}$ \\
\hline 2 & $\begin{array}{c}1.504 * * * \\
(0.318)\end{array}$ & $\begin{array}{l}2.349 * * * \\
(0.459)\end{array}$ & $\begin{array}{l}-0.339 \\
(0.373)\end{array}$ & $\begin{array}{c}0.620 * * * \\
(0.188)\end{array}$ & $\begin{array}{l}1.116^{* * *} \\
(0.208)\end{array}$ & $\begin{array}{l}-0.311 \\
(0.228)\end{array}$ & $\begin{array}{l}0.883^{* * *} \\
(0.252)\end{array}$ & $\begin{array}{c}1.232 * * * \\
(0.370)\end{array}$ & $\begin{array}{l}-0.027 \\
(0.268)\end{array}$ \\
\hline \multicolumn{10}{|c|}{$\begin{array}{l}\text { Panel C. Local estimates: Narrow-window sample } \\
\text { Order of polynomial }\end{array}$} \\
\hline 0 & $\begin{array}{c}0.335 \\
(0.272)\end{array}$ & $\begin{array}{c}0.750 \\
(0.484)\end{array}$ & $\begin{array}{l}-0.054 \\
(0.141)\end{array}$ & $\begin{array}{c}0.121 \\
(0.136)\end{array}$ & $\begin{array}{c}0.251 \\
(0.264)\end{array}$ & $\begin{array}{l}-0.006 \\
(0.113)\end{array}$ & $\begin{array}{c}0.214 \\
(0.162)\end{array}$ & $\begin{array}{l}0.499^{*} \\
(0.251)\end{array}$ & $\begin{array}{l}-0.048 \\
(0.046)\end{array}$ \\
\hline 1 & $\begin{array}{l}1.288^{* * *} \\
(0378)\end{array}$ & $\begin{array}{c}2.397 * * * \\
(0.750)\end{array}$ & $\begin{array}{l}-0.308 \\
(0.281)\end{array}$ & $\begin{array}{l}0.428^{*} \\
(0.228)\end{array}$ & $\begin{array}{c}1.012 \\
(0.350)\end{array}$ & $\begin{array}{l}-0.395 \\
(0.218)\end{array}$ & $\begin{array}{c}0.859 * * * \\
(0.223)\end{array}$ & $\begin{array}{l}1.384 * * \\
(0.457)\end{array}$ & $\begin{array}{c}0.087 \\
(0.105)\end{array}$ \\
\hline 2 & $\begin{array}{l}1.329 * * \\
(0.535)\end{array}$ & $\begin{array}{l}2.514 * * \\
(0.938)\end{array}$ & $\begin{array}{l}-1.116 \\
(0.379)\end{array}$ & $\begin{array}{c}0.228 \\
(0.432)\end{array}$ & $\begin{array}{c}0.841 \\
(0.502)\end{array}$ & $\begin{array}{c}-0.820^{* *} \\
(0.305)\end{array}$ & $\begin{array}{c}1.101 * * * \\
(0.277)\end{array}$ & $\begin{array}{l}1.672 * * \\
(0.607)\end{array}$ & $\begin{array}{l}-0.295 \\
(0.180)\end{array}$ \\
\hline
\end{tabular}

Notes: The table shows the estimates of the coefficients $\beta_{k}$ of model (2) using investment over pre-program capital as outcome variables. Number of observations (firms) is 357 in Panel A; 171 in
Panel B; 115 in Panel C. Pre-program capital used as scaling variable is the sum of tangible and intangible assets taken from the balance sheet data. For further details seethe notes to Tables 3-5. 
RESULTS OF KERNEL REGRESSIONS

INVESTMENT AND REIMBURSABLE COSTS NORMALIZED BY PRE-PROGRAM SALES

\begin{tabular}{|c|c|c|c|c|c|c|c|c|c|c|c|c|c|c|c|}
\hline & \multicolumn{5}{|c|}{ All firms } & \multicolumn{5}{|c|}{ Small firms } & \multicolumn{5}{|c|}{ Large firms } \\
\hline & $\begin{array}{c}\text { Total } \\
\text { investment }\end{array}$ & $\begin{array}{c}\text { Tangible } \\
\text { investment }\end{array}$ & $\begin{array}{l}\text { Intangible } \\
\text { investment }\end{array}$ & $\begin{array}{l}\text { Labor } \\
\text { costs }\end{array}$ & $\begin{array}{l}\text { Service } \\
\text { costs }\end{array}$ & $\begin{array}{c}\text { Total } \\
\text { investment }\end{array}$ & $\begin{array}{c}\text { Tangible } \\
\text { investment }\end{array}$ & $\begin{array}{l}\text { Intangible } \\
\text { investment }\end{array}$ & $\begin{array}{l}\text { Labor } \\
\text { costs }\end{array}$ & $\begin{array}{l}\text { Service } \\
\text { costs }\end{array}$ & $\begin{array}{c}\text { Total } \\
\text { investment }\end{array}$ & $\begin{array}{c}\text { Tangible } \\
\text { investment }\end{array}$ & $\begin{array}{l}\text { Intangible } \\
\text { investment }\end{array}$ & $\begin{array}{l}\text { Labor } \\
\text { costs }\end{array}$ & $\begin{array}{l}\text { Service } \\
\text { costs }\end{array}$ \\
\hline \multicolumn{16}{|c|}{$\begin{array}{l}\text { Panel } A \text {. Bandwidth }=30 \\
\text { Order of polynomial }\end{array}$} \\
\hline 0 & $\begin{array}{c}0.014 \\
(0.014)\end{array}$ & $\begin{array}{c}0.009 \\
(0.012)\end{array}$ & $\begin{array}{c}0.004 \\
(0.008)\end{array}$ & $\begin{array}{l}-0.051 \\
(0.057)\end{array}$ & $\begin{array}{l}-0.077 \\
(0.053)\end{array}$ & $\begin{array}{c}0.048 * * * \\
(0.016)\end{array}$ & $\begin{array}{l}0.026 * * \\
(0.013)\end{array}$ & $\begin{array}{l}0.022 * * \\
(0.011)\end{array}$ & $\begin{array}{l}-0.008 \\
(0.056)\end{array}$ & $\begin{array}{l}-0.061 \\
(0.102)\end{array}$ & $\begin{array}{l}-0.021 \\
(0.018)\end{array}$ & $\begin{array}{l}-0.010 \\
(0.021)\end{array}$ & $\begin{array}{l}-0.012 \\
(0.008)\end{array}$ & $\begin{array}{l}-0.086 \\
(0.091)\end{array}$ & $\begin{array}{c}-0.045 \\
(0.088)\end{array}$ \\
\hline 1 & $\begin{array}{l}0.041^{*} \\
(0.022)\end{array}$ & $\begin{array}{c}0.024 \\
(0.016)\end{array}$ & $\begin{array}{c}0.017 \\
(0.013)\end{array}$ & $\begin{array}{l}-0.059 \\
(0.089)\end{array}$ & $\begin{array}{c}0.029 \\
(0.089)\end{array}$ & $\begin{array}{c}0.081 * * * \\
(0.030)\end{array}$ & $\begin{array}{l}0.045^{* *} \\
(0.022)\end{array}$ & $\begin{array}{l}0.035^{*} \\
(0.021)\end{array}$ & $\begin{array}{l}-0.067 \\
(0.095)\end{array}$ & $\begin{array}{c}0.026 \\
(0.150)\end{array}$ & $\begin{array}{l}-0.011 \\
(0.031)\end{array}$ & $\begin{array}{l}-0.007 \\
(0.027)\end{array}$ & $\begin{array}{l}-0.003 \\
(0.012)\end{array}$ & $\begin{array}{l}-0.058 \\
(0.153)\end{array}$ & $\begin{array}{c}0.025 \\
(0.151)\end{array}$ \\
\hline 2 & $\begin{array}{c}0.047 \\
(0.031)\end{array}$ & $\begin{array}{c}0.022 \\
(0.023)\end{array}$ & $\begin{array}{c}0.024 \\
(0.018)\end{array}$ & $\begin{array}{c}-0.173 \\
(0.0143)\end{array}$ & $\begin{array}{l}-0.015 \\
(0.149)\end{array}$ & $\begin{array}{c}0.103^{* * *} \\
(0.042)\end{array}$ & $\begin{array}{c}0.057 * * * \\
(0.019)\end{array}$ & $\begin{array}{c}0.046 \\
(0.030)\end{array}$ & $\begin{array}{l}-0.082 \\
(0.175)\end{array}$ & $\begin{array}{c}0.092 \\
(0.261)\end{array}$ & $\begin{array}{l}-0.013 \\
(0.047)\end{array}$ & $\begin{array}{l}-0.011 \\
(0.032)\end{array}$ & $\begin{array}{l}-0.001 \\
(0.017)\end{array}$ & $\begin{array}{l}-0.274 \\
(0.234)\end{array}$ & $\begin{array}{l}-0.116 \\
(0.231)\end{array}$ \\
\hline 3 & $\begin{array}{c}0.066 \\
(0.051)\end{array}$ & $\begin{array}{c}0.024 \\
(0.051)\end{array}$ & $\begin{array}{c}0.042 \\
(0.029)\end{array}$ & $\begin{array}{l}-0.375 \\
(0.229)\end{array}$ & $\begin{array}{l}-0.056 \\
(0.224)\end{array}$ & $\begin{array}{c}0.148 \\
(0.116)\end{array}$ & $\begin{array}{c}0.079 \\
(0.107)\end{array}$ & $\begin{array}{c}0.069 \\
(0.064)\end{array}$ & $\begin{array}{l}-0.236 \\
(0.178)\end{array}$ & $\begin{array}{c}0.211 \\
(0.457)\end{array}$ & $\begin{array}{l}-0.026 \\
(0.092)\end{array}$ & $\begin{array}{l}-0.033 \\
(0.063)\end{array}$ & $\begin{array}{c}0.007 \\
(0.039)\end{array}$ & $\begin{array}{l}-0.571^{*} \\
(0.327)\end{array}$ & $\begin{array}{l}-0.540 \\
(0.374)\end{array}$ \\
\hline \multicolumn{16}{|c|}{$\begin{array}{l}\text { Panel B. Bandwidth }=15 \\
\text { Order of polynomial }\end{array}$} \\
\hline 0 & $\begin{array}{c}0.018 \\
(0.014)\end{array}$ & $\begin{array}{c}0.013 \\
(0.012)\end{array}$ & $\begin{array}{c}0.005 \\
(0.008)\end{array}$ & $\begin{array}{l}-0.051 \\
(0.063)\end{array}$ & $\begin{array}{l}-0.049 \\
(0.053)\end{array}$ & $\begin{array}{c}0.057^{* * *} \\
(0.017)\end{array}$ & $\begin{array}{l}0.034^{* *} \\
(0.012)\end{array}$ & $\begin{array}{l}0.022^{*} \\
(0.012)\end{array}$ & $\begin{array}{l}-0.023 \\
(0.065)\end{array}$ & $\begin{array}{l}-0.047 \\
(0.118)\end{array}$ & $\begin{array}{l}-0.020 \\
(0.018)\end{array}$ & $\begin{array}{l}-0.109 \\
(0.022)\end{array}$ & $\begin{array}{l}-0.009 \\
(0.008)\end{array}$ & $\begin{array}{l}-0.070 \\
(0.089)\end{array}$ & $\begin{array}{c}-0.013 \\
(0.091)\end{array}$ \\
\hline 1 & $\begin{array}{l}0.047^{*} \\
(0.025)\end{array}$ & $\begin{array}{c}0.024 \\
(0.019)\end{array}$ & $\begin{array}{l}0.023^{*} \\
(0.013)\end{array}$ & $\begin{array}{l}-0.142 \\
(0.091)\end{array}$ & $\begin{array}{c}-0.009 \\
(0.096)\end{array}$ & $\begin{array}{c}0.102^{* * * *} \\
(0.030)\end{array}$ & $\begin{array}{l}0.054^{* *} \\
(0.024)\end{array}$ & $\begin{array}{l}0.048^{* *} \\
(0.021)\end{array}$ & $\begin{array}{l}-0.087 \\
(0.092)\end{array}$ & $\begin{array}{c}0.081 \\
(0.161)\end{array}$ & $\begin{array}{l}-0.012 \\
(0.036)\end{array}$ & $\begin{array}{l}-0.007 \\
(0.028)\end{array}$ & $\begin{array}{l}-0.004 \\
(0.012)\end{array}$ & $\begin{array}{l}-0.192 \\
(0.169)\end{array}$ & $\begin{array}{c}-0.066 \\
(0.174)\end{array}$ \\
\hline 2 & $\begin{array}{c}0.058 \\
(0.043)\end{array}$ & $\begin{array}{c}0.020 \\
(0.032)\end{array}$ & $\begin{array}{c}0.038 \\
(0.023)\end{array}$ & $\begin{array}{c}-0.256 \\
(0.158)\end{array}$ & $\begin{array}{l}-0.010 \\
(0.153)\end{array}$ & $\begin{array}{c}0.135 * * * \\
(0.043)\end{array}$ & $\begin{array}{c}0.075 * * * \\
(0.019)\end{array}$ & $\begin{array}{c}0.060 \\
(0.038)\end{array}$ & $\begin{array}{c}-0.136 \\
(0.183)\end{array}$ & $\begin{array}{c}0.149 \\
(0.339)\end{array}$ & $\begin{array}{c}0.026 \\
(0.077)\end{array}$ & $\begin{array}{l}-0.039 \\
(0.049)\end{array}$ & $\begin{array}{c}0.013 \\
(0.027)\end{array}$ & $\begin{array}{l}-0.419 \\
(0.282)\end{array}$ & $\begin{array}{c}-0.284 \\
(0.303)\end{array}$ \\
\hline 3 & $\begin{array}{c}0.044 \\
(0.076)\end{array}$ & $\begin{array}{l}-0.010 \\
(0.059)\end{array}$ & $\begin{array}{c}0.055 \\
(0.034)\end{array}$ & $\begin{array}{l}-0.101 \\
(0.233)\end{array}$ & $\begin{array}{c}0.152 \\
(0.225)\end{array}$ & $\begin{array}{c}0.148 \\
(0.126)\end{array}$ & $\begin{array}{c}0.053 \\
(0.113)\end{array}$ & $\begin{array}{c}0.095 \\
(0.081)\end{array}$ & $\begin{array}{l}-0.024 \\
(0.225)\end{array}$ & $\begin{array}{c}0.292 \\
(0.715)\end{array}$ & $\begin{array}{c}-0.069 \\
(0.168)\end{array}$ & $\begin{array}{l}-0.073 \\
(0.129)\end{array}$ & $\begin{array}{c}0.004 \\
(0.056)\end{array}$ & $\begin{array}{l}-0.178 \\
(0.516)\end{array}$ & $\begin{array}{c}-0.228 \\
(0.587)\end{array}$ \\
\hline
\end{tabular}

Notes: The table reports the differences of the outcome variable between recipient and non-recipient firms estimated at the cut-off score (score=75). All the variables are accumulated over the first 3 years after the assignment (including that of the assignment) and scaled by sales in the pre-assignment year. We estimated the model using the Epanechnikov kernel combined with two bandwidths $( \pm 30$ and \pm 15$)$ and various polynomials. The full sample includes 341 firms in panel A and 271 in panel B. Bootstrapped standard errors (100 replications) clustered by score in round brackets. Polynomial of order 0 is the difference in mean between treated and untreated. Small (large) firms are those with value added below (above) the median.

$*^{*}, * * * *$ : significant at 10 percent, 5 percent, 1 percent, respectively. 
Outcome variable: Log (Wages)

\begin{tabular}{|c|c|c|c|c|c|c|}
\hline & All firms & Small firms & Large firms & All firms & Small firms & Large firms \\
\hline \multicolumn{7}{|c|}{$\begin{array}{l}\text { Panel B1. Bandwidth }=30 \\
\text { Order of polynomial }\end{array}$} \\
\hline 0 & $\begin{array}{l}0.253^{*} \\
(0.146)\end{array}$ & $\begin{array}{l}0.208^{*} \\
(0.113)\end{array}$ & $\begin{array}{l}0.243^{*} \\
(0.132)\end{array}$ & $\begin{array}{c}0.057 * * * \\
(0.022)\end{array}$ & $\begin{array}{l}-0.012 \\
(0.064)\end{array}$ & $\begin{array}{c}0.090^{* * *} \\
(0.028)\end{array}$ \\
\hline 1 & $\begin{array}{l}-0.054 \\
(0.264)\end{array}$ & $\begin{array}{l}0.298 \\
(0.394)\end{array}$ & $\begin{array}{l}-0.021 \\
(0.214)\end{array}$ & $\begin{array}{c}0.024 \\
(0.036)\end{array}$ & $\begin{array}{l}-0.025 \\
(0.079)\end{array}$ & $\begin{array}{c}0.061 \\
(0.039)\end{array}$ \\
\hline 2 & $\begin{array}{l}0.523 * \\
(0.303)\end{array}$ & $\begin{array}{c}0.793 \\
(0.765)\end{array}$ & $\begin{array}{l}-0.165 \\
(0.332)\end{array}$ & $\begin{array}{c}0.029 \\
(0.054)\end{array}$ & $\begin{array}{l}-0.007 \\
(0.779)\end{array}$ & $\begin{array}{c}0.031 \\
(0.072)\end{array}$ \\
\hline 3 & $\begin{array}{c}0.344 \\
(0.450)\end{array}$ & $\begin{array}{c}0.319 \\
(1.199)\end{array}$ & $\begin{array}{l}-0.138 \\
(0.619)\end{array}$ & $\begin{array}{c}0.013 \\
(0.165)\end{array}$ & $\begin{array}{l}0.015 \\
(0.275)\end{array}$ & $\begin{array}{l}-0.000 \\
(0.179)\end{array}$ \\
\hline \multicolumn{7}{|c|}{$\begin{array}{l}\text { Panel B2. Bandwidth }=15 \\
\text { Order of polynomial }\end{array}$} \\
\hline 0 & $\begin{array}{c}0.213 \\
(0.135)\end{array}$ & $\begin{array}{l}0.191 * \\
(0.109)\end{array}$ & $\begin{array}{l}0.245^{*} \\
(0.129)\end{array}$ & $\begin{array}{l}0.058 * * \\
(0.028)\end{array}$ & $\begin{array}{l}-0.015 \\
(0.013)\end{array}$ & $\begin{array}{c}0.094 * * * \\
(0.028)\end{array}$ \\
\hline 1 & $\begin{array}{c}0.186 \\
(0.251)\end{array}$ & $\begin{array}{c}0.505 \\
(0.393)\end{array}$ & $\begin{array}{l}-0.058 \\
(0.220)\end{array}$ & $\begin{array}{c}0.011 \\
(0.036)\end{array}$ & $\begin{array}{l}-0.017 \\
(0.131)\end{array}$ & $\begin{array}{c}0.032 \\
(0.041)\end{array}$ \\
\hline 2 & $\begin{array}{c}0.325 \\
(0.411)\end{array}$ & $\begin{array}{c}0.472 \\
(0.760)\end{array}$ & $\begin{array}{l}-0.267 \\
(0.478)\end{array}$ & $\begin{array}{c}0.023 \\
(0.074)\end{array}$ & $\begin{array}{l}-0.024 \\
(0.164)\end{array}$ & $\begin{array}{c}0.038 \\
(0.095)\end{array}$ \\
\hline \multicolumn{7}{|c|}{$\begin{array}{l}\text { Notes: The table reports the differences of the outcome variable between recipient and non-recipient firms estimated at the cut-off score } \\
\text { (score=75). Employment is accumulated over the first } 3 \text { years after the assignment (including that of the assignment). Polynomial of order } 0 \text { is } \\
\text { the difference in mean between treated and untreated. Small (large) firms are those with value added below (above) the median. } \\
\text { We estimated the model using the Epanechnikov kernel combined with two bandwidths ( } \pm 30 \text { and } \pm 15 \text { points around the cut-off) and various } \\
\text { polynomials. In panel B1 the number of observations (firms) is } 263 \text {; in panel B2 is } 271 \text {. Bootstrapped standard errors (100 replications) } \\
\text { clustered by score in round brackets. } \\
*, * * * * * \text { : significant at } 10 \text { percent, } 5 \text { percent, } 1 \text { percent, respectively. }\end{array}$} \\
\hline
\end{tabular}


Table B11

ROBUSTNESS: DISCONTINUITY OF COVARIATES

\begin{tabular}{|c|c|c|c|c|c|c|c|c|}
\hline & \multicolumn{2}{|c|}{ ROA } & \multicolumn{2}{|c|}{ Net worth assets/Debts } & \multicolumn{2}{|c|}{ Cash flow/Sales } & \multicolumn{2}{|c|}{ Interest costs/Debts } \\
\hline & Small & Large & Small & Large & Small & Large & Small & Large \\
\hline \multicolumn{9}{|c|}{$\begin{array}{l}\text { Panel A. Full sample } \\
\text { Order of polynomial }\end{array}$} \\
\hline 0 & $\begin{array}{c}0.139 \\
(1.575)\end{array}$ & $\begin{array}{c}0.317 \\
(1.288)\end{array}$ & $\begin{array}{c}0.042 \\
(0.109)\end{array}$ & $\begin{array}{c}0.018 \\
(0.087)\end{array}$ & $\begin{array}{c}0.015 \\
(0.018)\end{array}$ & $\begin{array}{c}0.006 \\
(0.008)\end{array}$ & $\begin{array}{l}-0.001 \\
(0.003)\end{array}$ & $\begin{array}{l}-0.001 \\
(0.002)\end{array}$ \\
\hline 1 & $\begin{array}{l}-1.777 \\
(2.329)\end{array}$ & $\begin{array}{l}-0.515 \\
(1.581)\end{array}$ & $\begin{array}{l}-0.223 \\
(0.149)\end{array}$ & $\begin{array}{c}0.035 \\
(0.133)\end{array}$ & $\begin{array}{l}-0.030 \\
(0.021)\end{array}$ & $\begin{array}{l}-0.004 \\
(0.009)\end{array}$ & $\begin{array}{l}-0.000 \\
(0.005)\end{array}$ & $\begin{array}{c}0.000 \\
(0.003)\end{array}$ \\
\hline 2 & $\begin{array}{l}-1.967 \\
(2.502)\end{array}$ & $\begin{array}{l}1.191 \\
(2.122)\end{array}$ & $\begin{array}{r}-0.387 * \\
(0.197)\end{array}$ & $\begin{array}{l}-0.132 \\
(0.196)\end{array}$ & $\begin{array}{l}-0.048 \\
(0.032)\end{array}$ & $\begin{array}{c}0.001 \\
(0.001)\end{array}$ & $\begin{array}{c}0.001 \\
(0.008)\end{array}$ & $\begin{array}{c}0.007 \\
(0.006)\end{array}$ \\
\hline \multicolumn{9}{|c|}{$\begin{array}{l}\text { Panel B. Local estimates: Wide-window sample } \\
\text { Order of polynomial }\end{array}$} \\
\hline 0 & $\begin{array}{l}-2.325 \\
(1.872)\end{array}$ & $\begin{array}{l}-0.635 \\
(1.196)\end{array}$ & $\begin{array}{l}-0.161 \\
(0.111)\end{array}$ & $\begin{array}{l}-0.046 \\
(0.098)\end{array}$ & $\begin{array}{l}-0.013 \\
(0.014)\end{array}$ & $\begin{array}{l}-0.002 \\
(0.008)\end{array}$ & $\begin{array}{c}0.001 \\
(0.004)\end{array}$ & $\begin{array}{c}0.001 \\
(0.003)\end{array}$ \\
\hline 1 & $\begin{array}{l}-0.494 \\
(2.456)\end{array}$ & $\begin{array}{c}1.172 \\
(2.098)\end{array}$ & $\begin{array}{l}-0.237 \\
(0.196)\end{array}$ & $\begin{array}{c}0.108 \\
(0.205)\end{array}$ & $\begin{array}{l}-0.032 \\
(0.025)\end{array}$ & $\begin{array}{c}0.005 \\
(0.011)\end{array}$ & $\begin{array}{l}-0.006 \\
(0.008)\end{array}$ & $\begin{array}{c}0.013 \\
(0.009)\end{array}$ \\
\hline 2 & $\begin{array}{c}3.592 \\
(4.446)\end{array}$ & $\begin{array}{c}1.513 \\
(4.495)\end{array}$ & $\begin{array}{l}-0.265 \\
(0.386)\end{array}$ & $\begin{array}{c}0.902 * * * \\
(0.240)\end{array}$ & $\begin{array}{c}0.006 \\
(0.032)\end{array}$ & $\begin{array}{c}0.000 \\
(0.028)\end{array}$ & $\begin{array}{l}-0.004 \\
(0.012)\end{array}$ & $\begin{array}{c}0.027 \\
(0.016)\end{array}$ \\
\hline \multicolumn{9}{|c|}{$\begin{array}{l}\text { Panel C. Local estimates: Narrow-window sample } \\
\text { Order of polynomial }\end{array}$} \\
\hline 0 & $\begin{array}{l}-1.357 \\
(1.192)\end{array}$ & $\begin{array}{c}0.596 \\
(1.084)\end{array}$ & $\begin{array}{l}-0.132 \\
(0.138)\end{array}$ & $\begin{array}{l}-0.020 \\
(0.123)\end{array}$ & $\begin{array}{l}-0.021 \\
(0.017)\end{array}$ & $\begin{array}{c}0.005 \\
(0.008)\end{array}$ & $\begin{array}{l}-0.002 \\
(0.006)\end{array}$ & $\begin{array}{c}0.003 \\
(0.004)\end{array}$ \\
\hline 1 & $\begin{array}{c}1.405 \\
(4.656)\end{array}$ & $\begin{array}{l}-1.349 \\
(3.804)\end{array}$ & $\begin{array}{l}-0.358 \\
(0.346)\end{array}$ & $\begin{array}{c}0.555^{* *} \\
(0.225)\end{array}$ & $\begin{array}{l}-0.002 \\
(0.028)\end{array}$ & $\begin{array}{l}-0.024 \\
(0.018)\end{array}$ & $\begin{array}{l}-0.010 \\
(0.013)\end{array}$ & $\begin{array}{c}0.021 \\
(0.016)\end{array}$ \\
\hline 2 & $\begin{array}{l}-8.457 \\
(5.410)\end{array}$ & $\begin{array}{l}11.978 \\
(3.701)\end{array}$ & $\begin{array}{l}-0.065 \\
(0.467)\end{array}$ & $\begin{array}{c}1.606^{* * *} \\
(0.382)\end{array}$ & $\begin{array}{c}0.016 \\
(0.064)\end{array}$ & $\begin{array}{c}0.032 \\
(0.023)\end{array}$ & $\begin{array}{c}0.007 \\
(0.023)\end{array}$ & $\begin{array}{c}0.023 \\
(0.013)\end{array}$ \\
\hline
\end{tabular}

Notes: The table shows the estimates of the coefficients $\beta_{k}$ of model (2) using different outcome variables. Number of observations (firms) is 357 in Panel A; 171 in Panel B; 115 in Panel C. Robust standard errors clustered by score are in round brackets. For further details see the Notes to table 5 .

$*, * *, * * *$ : significant at 10 percent, 5 percent, 1 percent, respectively. 
Table B12

ROBUSTNESS: TESTS FOR DISCONTINUITY IN THE PRE-PROGRAM PERIOD AND AT DIFFERENT CUT-OFF POINTS

Panel 1. Tests for discontinuity in the pre-program period

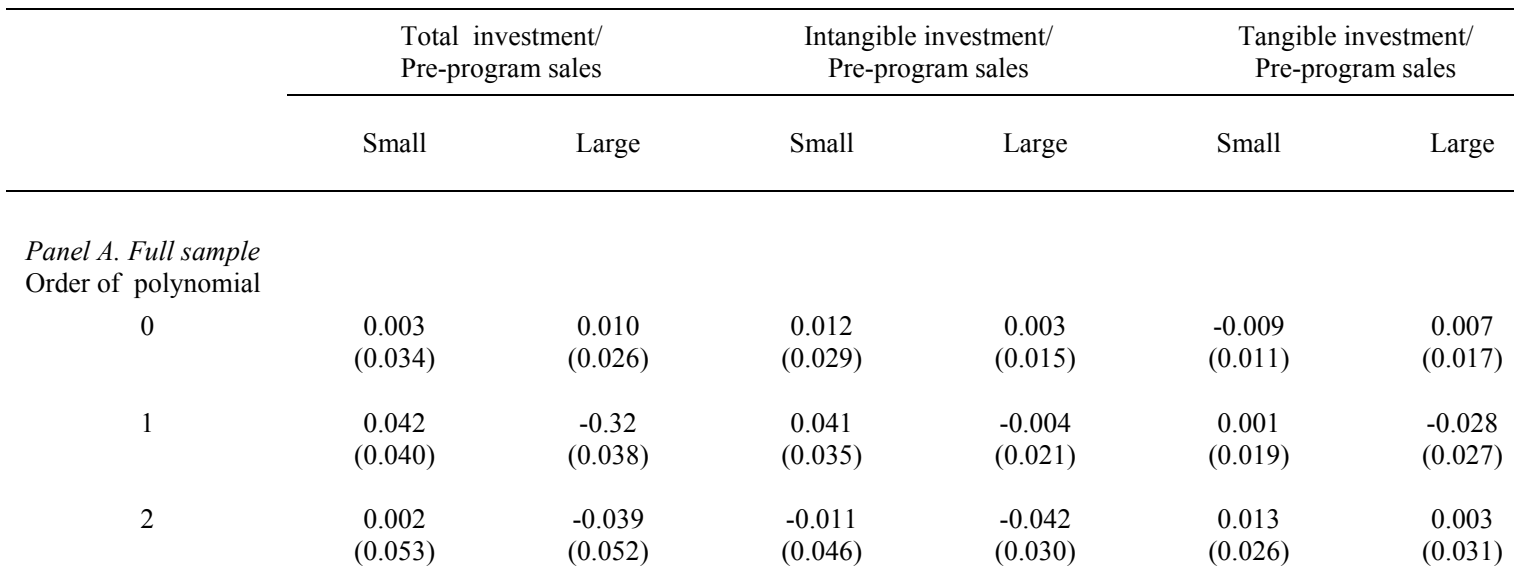

Panel B. Local estimates: Wide-window sample Order of polynomial

\begin{tabular}{|c|c|c|c|c|c|c|}
\hline 0 & $\begin{array}{c}0.022 \\
(0.034)\end{array}$ & $\begin{array}{c}-0.011 \\
(0.024)\end{array}$ & $\begin{array}{c}0.028 \\
(0.027)\end{array}$ & $\begin{array}{c}-0.004 \\
(0.017)\end{array}$ & $\begin{array}{c}-0.006 \\
(0.018)\end{array}$ & $\begin{array}{l}-0.006 \\
(0.011)\end{array}$ \\
\hline 1 & $\begin{array}{c}0.019 \\
(0.058)\end{array}$ & $\begin{array}{c}-0.011 \\
(0.043)\end{array}$ & $\begin{array}{l}-0.005 \\
(0.047)\end{array}$ & $\begin{array}{l}-0.008 \\
(0.030)\end{array}$ & $\begin{array}{c}0.025 \\
(0.035)\end{array}$ & $\begin{array}{c}-0.003 \\
(0.019)\end{array}$ \\
\hline 2 & $\begin{array}{l}-0.006 \\
(0.076)\end{array}$ & $\begin{array}{c}0.011 \\
(0.059)\end{array}$ & $\begin{array}{l}-0.014 \\
(0.060)\end{array}$ & $\begin{array}{c}0.013 \\
(0.043)\end{array}$ & $\begin{array}{c}0.008 \\
(0.036)\end{array}$ & $\begin{array}{l}-0.002 \\
(0.034)\end{array}$ \\
\hline
\end{tabular}

Panel C. Local estimates: Narrow-window sample Order of polynomial

\begin{tabular}{|c|c|c|c|c|c|c|}
\hline 0 & $\begin{array}{c}0.041 \\
(0.042)\end{array}$ & $\begin{array}{c}-0.008 \\
(0.030)\end{array}$ & $\begin{array}{c}0.026 \\
(0.036)\end{array}$ & $\begin{array}{c}-0.001 \\
(0.023)\end{array}$ & $\begin{array}{c}0.014 \\
(0.024)\end{array}$ & $\begin{array}{l}-0.006 \\
(0.013)\end{array}$ \\
\hline 1 & $\begin{array}{c}-0.024 \\
(0.096)\end{array}$ & $\begin{array}{l}-0.022 \\
(0.065)\end{array}$ & $\begin{array}{c}-0.019 \\
(0.083)\end{array}$ & $\begin{array}{c}-0.017 \\
(0.050)\end{array}$ & $\begin{array}{c}-0.004 \\
(0.039)\end{array}$ & $\begin{array}{l}-0.005 \\
(0.032)\end{array}$ \\
\hline 2 & $\begin{array}{c}-0.109 * \\
(0.059)\end{array}$ & $\begin{array}{c}0.056 \\
(0.059)\end{array}$ & $\begin{array}{l}-0.075 \\
(0.048)\end{array}$ & $\begin{array}{c}0.042 \\
(0.040)\end{array}$ & $\begin{array}{c}-0.033 \\
(0.020)\end{array}$ & $\begin{array}{c}0.014 \\
(0.025)\end{array}$ \\
\hline
\end{tabular}

Panel 2. F-Tests for discontinuities at different cut-off points

\begin{tabular}{|c|c|c|c|}
\hline Order of polynomial & $\begin{array}{l}\text { Total investment/ } \\
\text { Pre-program sales }\end{array}$ & $\begin{array}{l}\text { Total investment/ } \\
\text { Pre-program capital }\end{array}$ & $\begin{array}{l}\text { Total investment/ } \\
\text { Pre-program assets }\end{array}$ \\
\hline 0 & $\begin{array}{c}1.12 \\
(0.28)\end{array}$ & $\begin{array}{c}1.11 \\
(0.30)\end{array}$ & $\begin{array}{c}1.27 \\
(0.12)\end{array}$ \\
\hline 1 & $\begin{array}{c}1.06 \\
(0.37)\end{array}$ & $\begin{array}{c}1.02 \\
(0.44)\end{array}$ & $\begin{array}{c}1.26 \\
(0.14)\end{array}$ \\
\hline 2 & $\begin{array}{c}1.07 \\
(0.36)\end{array}$ & $\begin{array}{c}1.01 \\
(0.45)\end{array}$ & $\begin{array}{c}1.22 \\
(0.17)\end{array}$ \\
\hline
\end{tabular}

Notes: The first panel of the table shows the estimates of the coefficients $\beta_{k}$ of model (2) using investment of 2 years before the implementation of the program. Number of observations (firms) is 346 in Panel A; 166 in Panel B; 113 in Panel C. Robust standard errors clustered by score are in round brackets. The second panel shows the F- tests for the null hypothesis that a full set of score dummies interacted with the small-firms dummy included in the model (2) are equal to zero. The full sample of 357 firms has been used. P-value are in round brackets. For further details see the notes to Tables 3 and 5. 